TP Periodica Polytechnica Chemical Engineering

60(1), pp. 49-53, 2016

DOI: $10.3311 /$ PPch. 8500

Creative Commons Attribution (i)

RESEARCH ARTICLE

\section{Microencapsulation Analysis Based on Membrane Technology: Basic Research of Spherical, Solid Precursor Microcapsule Production}

\author{
Krisztina Albert $^{1 *}$, Csilla Tóth ${ }^{1}$, Balázs Verasztó ${ }^{1}$, \\ Gyula Vatai ${ }^{1}$, András Koris ${ }^{1}$
}

Received 14 August 2015; accepted after revision 25 October 2015

\begin{abstract}
The objective of the proposed investigation was to synthesis of precursor oil-carbohydrate microcapsule, suspended in water by membrane emulsification technology. To select the carrier material of precursor microcapsules, four different carbohydrates (maltodextrin, hydroxypropyl cellulose, potato starch and corn starch) were tested. Preliminary, zeta-potential, molecular weight and particle size of individual carbohydrate were estimated by Malvern Zetasizer instrument. Based on the results of the preliminary characterizations, maltodextrin was selected to carry out subsequent experiments. Maltodextrin suspended in oil and water were considered as dispersed phase and continuous phase respectively. An attempt has been made to remove the water, as well as to recover the microcapsules. It was observed that average particle size of synthesized microcapsule is $6.9 \mu \mathrm{m}$ and stability of the microcapsule is at least 6 weeks.
\end{abstract}

\section{Keywords}

Membrane emulsification, Microencapsulation, Sunflower oil, maltodextrin, Average particle size of microcapsule, Stability of microcapsule

\footnotetext{
${ }^{1}$ Department of Food Engineering, Faculty of Food Science, Corvinus University of Budapest

H-1118 Budapest, Ménesi st. 44., Hungary

*Corresponding author, e-mail: krisztina.albert1986@gmail.com
}

\section{Introduction}

The emergence of biotechnology is a boon to the industrial biological research and academics, which possess a revolutionary challenge for the production of valuable biomolecules. Presently, intellectual uprising with new visions and hopes boost up the renovation and development of equipment and process technology for the manufacturing of various products, such as food-beverages, pharmaceuticals from biological sources.

In bio-pharmaceutical, chemical, cosmetic, foods and printing industries, encapsulation technology has been practiced from a long prior [1]. In this technique, one material or a mixture of materials is coated with or entrapped within another material or system. The coated material is called active or core material, and the coating material is called shell or wall material or carrier or encapsulant [2]. Microencapsulation is a process for coating individual particles or droplets with a continuous film to produce capsules in a micrometer to millimeter in size aiming to protect the active material against undesirable reactions like oxidation [3].

In addition to the traditional methods of encapsulation (spray drying, extrusion, coacervation, fluid bed drying, spray cooling/chilling and molecular inclusion), emerging technology is membrane emulsification. The membrane emulsification or membrane contactor technique is a suitable method for producing several types of nanodispersions [4-6].

Membrane emulsification involves the permeation of dispersed phase into a tangentially moving continuous phase through a porous channel of membrane. The organic phase is pressed through the membrane pores and produce small droplets in aqueous phase [6].

For microencapsulation of oil, physical and chemical properties of carrier material and combination of different carrier materials affect the encapsulation efficiency and storage stability [7]. The process for encapsulation of oil consists of two steps: firstly, emulsification of core material with a dense solution of carrier materials and secondly, dry or cool the emulsion for recovery of encapsulated product [8].

In the present investigation, authors tried their best to prepare precursor microcapsule by membrane emulsification technology in advance and secondly recover the microcapsules 
from the emulsion by vacuum evaporation. To achieve this goal following steps were performed:

1. Preliminary characterization of different carbohydrates (zeta-potential, molecular weight and particle size) to select the most suitable carrier for synthesis of microcapsule.

2. Synthesis of solid oil microcapsules precursor by crossflow membrane emulsification procedure.

3. Studies on effect of surfactants for $\mathrm{o} / \mathrm{w}$ emulsification.

4. Recovery of the microcapsules from emulsion solution.

5. Characterization (size, shape and morphology of microcapsules, distribution) of synthesized microcapsules.

6. Studies on stability of microcapsules.

\section{Materials and methods}

\subsection{Materials}

Prior to the experiments, all the chemicals were procured from Sigma Aldrich, Germany. Commercial grade sunflower seed oil was purchased from local market in and around Budapest, Hungary.

\subsection{Cross-flow membrane emulsification apparatus}

Cross flow membrane module was used for membrane emulsification process (Fig. 1). The apparatus included two manometers at opposite two end of membrane to measure the pressure drop along the membrane. The dispersed phase pressure was guaranteed by compressed air with compressor and it was injected from the outer surface of the membrane. Peristaltic pump and recirculation channel were fitted to recirculate the continuous phase. A rotameter was placed at the exit side of the membrane for measuring the flow rate of continuous phase.

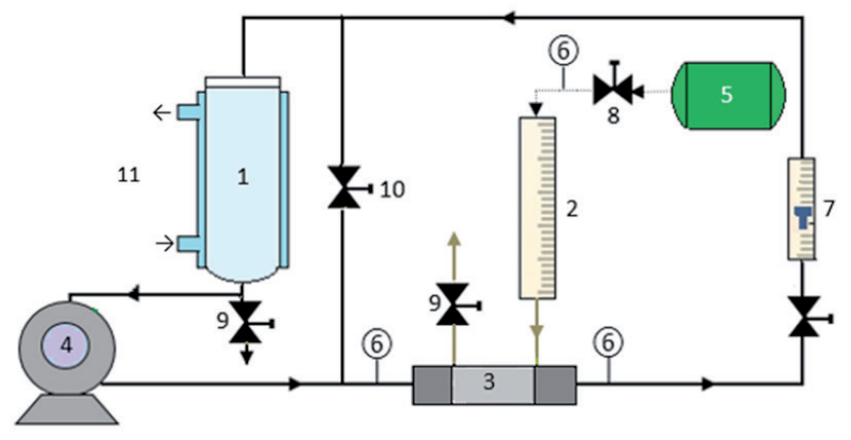

Fig. 1 Experimental set-up for the cross-flow membrane emulsification process; (1) Continuous phase tank, (2) Graduated disperse phase tank, (3) Membrane module, (4) Peristaltic pump, (5) Compressor, (6) Pressure meter, (7) Rotameter, (8) Pressure controller, (9) Valve on drain, (10) Valve, (11) Heating / Cooling (Thermostat)

\subsection{Characterization of carrier materials}

For the selection of best carrier material, four different carbohydrates, such as maltodextrin, hydroxypropyl cellulose, potato starch and corn starch were characterized. Preliminary characterizations, such as molecular weight, particle (molecule) size and zeta-potential of individual carbohydrate were studied by Malvern Zetasizer analytical instrument (Zetasizer Nano-ZS, Malvern, England). The zeta-potential is a key indicator of the stability of colloidal dispersions. It refers the ability of aggregation of the dispersed particles. Therefore, to estimate the zeta-potential of individual carbohydrate, different concentrations ranging from $10^{-5}$ to $10^{-1}(\mathrm{w} / \mathrm{w} \%)$ at $\mathrm{pH}=$ 4 were used. The subsequent experiments were performed by selected most suitable carbohydrate, used as carrier material.

\subsection{Microencapsulation by membrane emulsification}

In the present investigation, precursor microcapsules were prepared by tubular ceramic porous membrane, pore size $1.4 \mu \mathrm{m}$. The membrane material was $\alpha$ alumina and the active membrane surface area was $50 \mathrm{~cm}^{2}$. Cross-flow operation was adopted for emulsion production. Initially, selected carbohydrate (maltodextrin) was mixed with sunflower seed oil (50 $\mathrm{m} \%$ ). This mixture was considered as dispersed phase and it was pressed through the membrane pores under pressure. The continuous phase was distilled water, it contained $2 \%$ of Tween 80 emulsifier for easier emulsification and drop stability. The driving force was 4 bars; the flux was $4 \mathrm{~L} / \mathrm{m}^{2} \mathrm{~h}$. The direction of flow of dispersed phase was tangential with the membrane surface. Under the pressure, the dispersed phase formed as a drop at the pores opening in the membrane surface and subsequently those were detached by the cross-flowing continuous phase. For shaping the capsules the membrane pores were responsible.

\subsection{Recovery and analysis of microcapsules}

To recover the capsules from the emulsion, a rotary vacuum evaporator was used (operating pressure -0.5 bar, temperature $\left.84-86^{\circ} \mathrm{C}\right)$.

After recovery of microcapsules, the size, shape and morphology of microcapsules were analysed by optical microscope (DELTA OPTICAL, USA) and associated microscope image reader.

\section{Results and discussion}

\subsection{Characterization of carrier materials}

\subsubsection{Zeta-potential}

During the preliminary characterization, zeta potential was measured by Malvern Zetasizer apparatus. The zeta potential is a key indicator of the stability of colloidal dispersions. It refers to the ability of aggregation of the dispersed particles. As a rule of thumb, suspensions with zeta-potential above $30 \mathrm{mV}$ and below $-30 \mathrm{mV}$ are physically stable [9]. As the zeta-potential of any material depends on its concentration in the solution, therefore different concentrations of carbohydrate were prepared individually to estimate their zeta-potential (dilution range $10^{-5}$ to $10^{-1} \%$ ). For this analysis we used bent capillary tube cell.

The results of the zeta-potential for different carbohydrates are summarized in Fig. 2. In the figure, the values of zeta-potential 
of different carbohydrates have been plotted as a function of their concentrations and average values for each concentration are indicated. From the Fig. 2a, it is observed that average values of zeta- potential of maltodextrin is $-7.04 \mathrm{mV}$ for the concentration of $10^{-1} \%(\mathrm{w} / \mathrm{w})$ which signifies that for the concentration $10^{-1} \%(\mathrm{w} / \mathrm{w})$ the presence of maltodextrin in the solution is unstable. At lowest concentration $\left(10^{-5} \%\right)$ the average value of the zeta-potential of maltodextrin decreases to $-27.26 \mathrm{mV}$.
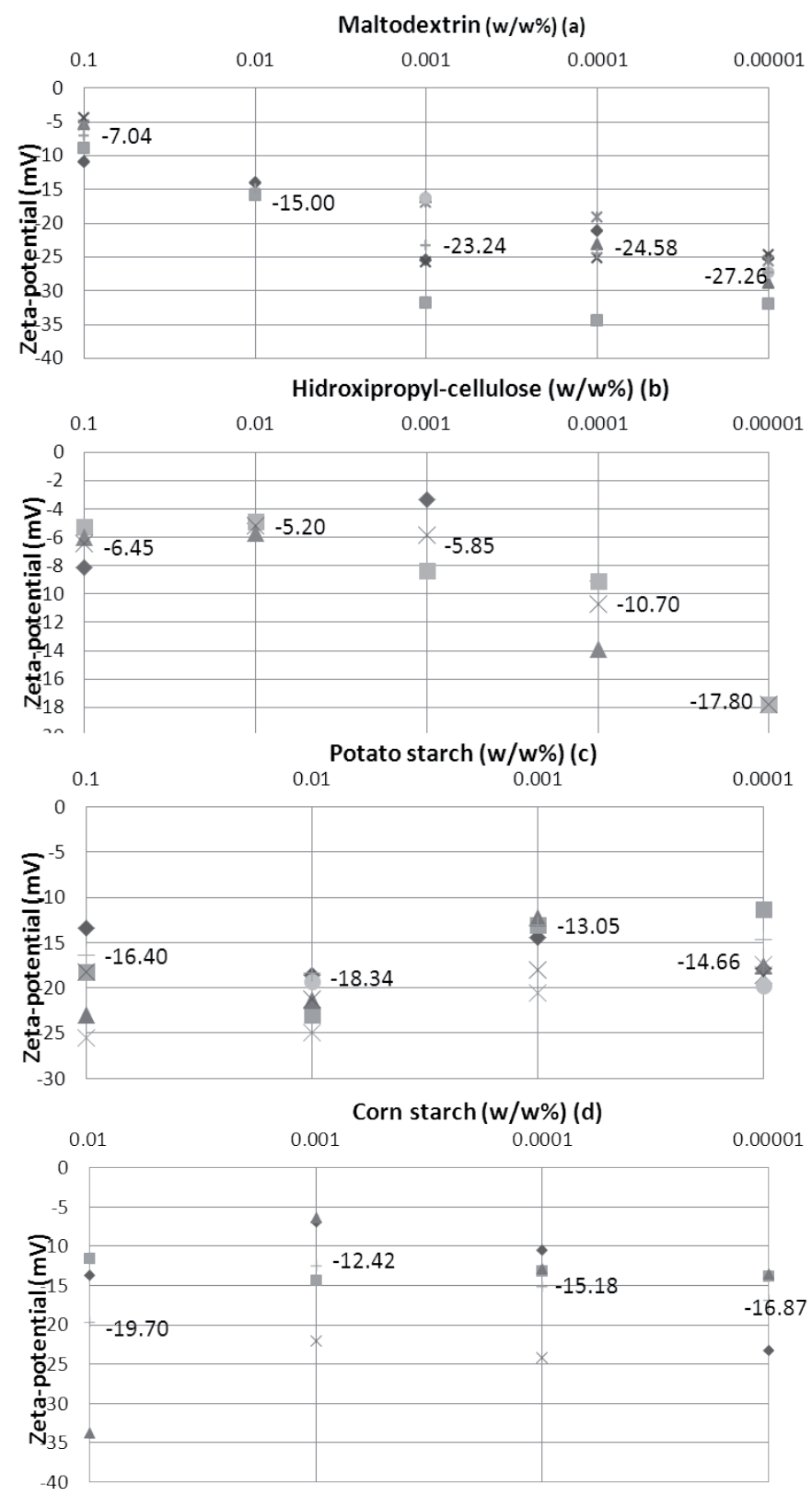

Fig. 2 Results of the zeta-potential for different concentrations of carrier carbohydrates, (a) maltodextrin, (b) hydroxypropyl cellulose, (c) potato starch, (d) corn starch

From the figure, it is notified that with increase the dilution, the average value of zeta-potential is turned toward $-30 \mathrm{mV}$, which signify that high dilution (low concentration of maltodextrin) has a positive impact on stability of maltodextrin in the solution (Fig. 2a).
The zeta-potential of hydroxypropyl cellulose at different dilution levels is portrayed in Fig. 2b. From the figure, it is observed that average values of zeta-potential of hydroxypropyl cellulose are lower than maltodextrin in the dilution range $10^{-5}$ to $10^{-1} \%$, which signify that stability of hydroxypropyl cellulose in the solution is low compared with maltodextrin in the entire dilution range of maltodextrin. Similar with the previous observation, it is also notified that with increase the dilution level of hydroxypropyl cellulose, the stability of hydroxypropyl cellulose in the solution increases. The figure portrayed that dilution has smaller effect on zeta-potential for hydroxypropyl cellulose than maltodextrin.

In Fig. 2c and Fig. 2d, the effects of dilution on zeta-potential of potato starch and corn starch respectively are depicted. The concentration of potato starch in the solution was varied ranging from $10^{-4}$ to $10^{-1} \%$. From the figure it is observed that the dilution has no effect on zeta-potential, as well as stability of potato starch in solution at above mentioned concentration range. In case of corn starch, concentration of corn starch in the solution was varied in the range of $10^{-5}$ to $10^{-2} \%$. Similar type of observation has been notified in this case also. From the figures, it may say that both of the carbohydrates in above mentioned concentration range are in unstable region.

\subsubsection{Molecular weight}

The molecular weights of each carbohydrate were estimated by Malvern Zetasizer. Debye plot have been developed for estimation of molecular weight of each carbohydrate and results are reported in Table 1. The coefficients of determination $\left(\mathrm{R}^{2}\right)$ of the measurements are indicated.

Table 1 Molecular weights of different carbohydrates

\begin{tabular}{lrc}
\hline Carbohydrate & $\mathrm{M}_{\mathrm{D}}(\mathrm{kDa})$ & \multicolumn{1}{c}{$\mathrm{R}^{2}$} \\
\hline Maltodextrin & $59 \pm 6$ & 1.00 \\
Hydroxypropyl cellulose & $1020 \pm 440$ & 0.97 \\
Potato starch & $1020 \pm 120$ & 1.00 \\
Corn starch & $496 \pm 97$ & 0.87 \\
\hline
\end{tabular}

From the Table, it is observed that maltodextrin has a lower molecular weight compare to other carbohydrates.

\subsubsection{Particle size}

The particle sizes of each carbohydrate were measured by Malvern Zetasizer. Table 2 depicts the average particle sizes of each carbohydrate and polydispersity index (PDI). From the table it is observed that the particle size of maltodextrin is lowest than other carbohydrates.

Wrapping up all the information, reported above, it may be said that hydroxypropyl cellulose and maltodextrin molecules can pass through the nominal diameter membrane pores 
$(1.4 \mu \mathrm{m})$. It was found from the experimental observation that hydroxypropyl cellulose creates gel layer on the vicinity of membrane which leads to membrane fouling. Therefore, maltodextrin was selected instead of hydroxypropyl cellulose as a carrier material of microcapsules.

Table 2 Particle sizes of different carbohydrates

\begin{tabular}{lccc}
\hline Material name & Concentration $(\mathrm{g} / \mathrm{L})$ & $\mathrm{d}_{\text {mean }}(\mathrm{nm})$ & $\mathrm{PDI}(-)$ \\
\hline Maltodextrin & $0.1-0.01$ & 170 & 0.253 \\
Hydroxypropyl cellulose & 0.01 & 260 & 0.203 \\
Potato starch & - & 3269 & 0.674 \\
Corn starch & $0.01-0.001$ & 1912 & 0.742 \\
\hline
\end{tabular}

\subsection{Characterization of synthesized microcapsule}

Microcapsules were prepared according to the method; described in Section 2.4. After synthesis of microcapsule by membrane based technology, vacuum evaporation was used to remove the water. It was observed that the final product is highly viscous, stable, white paste. Therefore, it may be said that the process is acceptable to produce solid precursor microcapsules. In Fig. 3, the photograph of final product (precursor microcapsule after removing the water) is depicted.

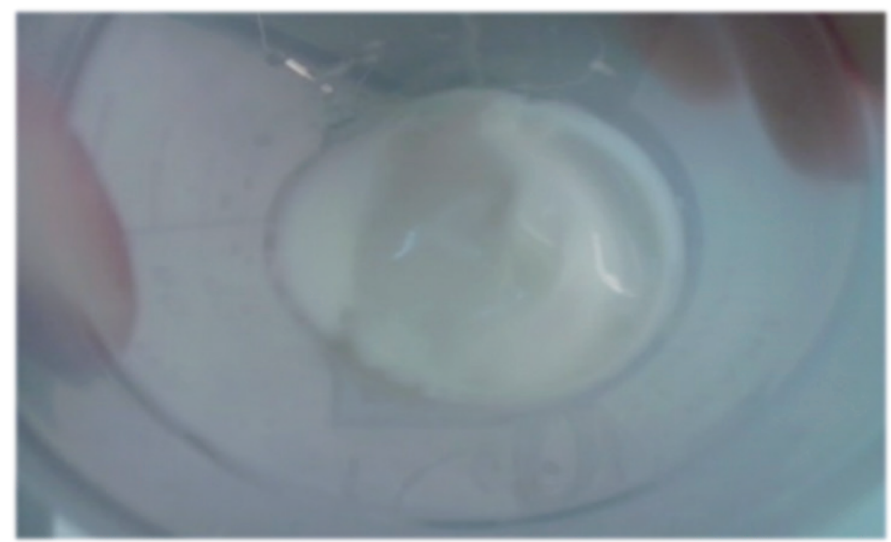

Fig. 3 Photograph of precursor microcapsule after removing the water

The diameters of the microcapsules were measured by optical microscope and the size frequency was plotted as a function of particle size (Fig. 4).

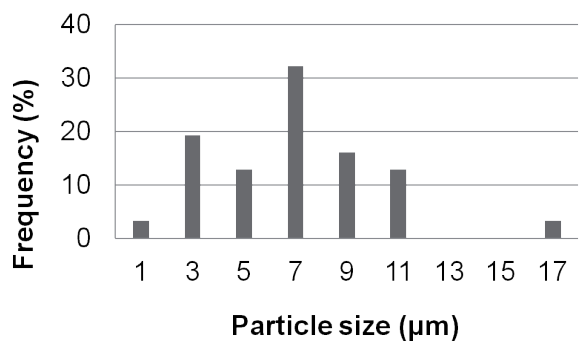

Fig. 4 Size distribution of the capsules
From the Fig. 4, it is observed that size of most (32\%) of synthesized particle is $7 \mu \mathrm{m}$. Also some of them, $3 \mu \mathrm{m}$ and $9 \mu \mathrm{m}$ are $18 \%$ and $15 \%$ respectively. From the above experimental result, the average size of microcapsules is calculated and that is $6.9 \mu \mathrm{m}$.

In the year 2000, Joscelyne with his co-worker reported that for membrane emulsification, the size of synthesized emulsify particle might be 2-10 times greater than the pore size of membrane. Present investigators used microporous membrane, pore size $1.4 \mu \mathrm{m}$ and they found that the average size of synthesized microcapsules is $6.9 \mu \mathrm{m}$, which supports the conclusion provided by previous mentioned research group [10].

\subsection{Stability analysis of synthesized microcapsule}

Experiments were conducted to understand the effect of surfactant (Tween 80) on microencapsulation formation and their stability. After preparing the microcapsule by membrane emulsification technology, the samples were stored at room temperature $\left(20^{\circ} \mathrm{C}\right)$ for six weeks and after that their morphology, size and shape have been analysed under the microscope. It was observed that the stability of microcapsule is $2-3 \mathrm{hr}$ when experiment was performed without surfactant. Contradictorily, it was observed that the surfactant provides the stability of microcapsule. The shape, size and morphology of synthesized microcapsule, developed in presence of surfactant, remain unchanged after their storage for 6 weeks also. Figure 5 shows the size and morphology of synthesized precursor microcapsules before and after their storage.

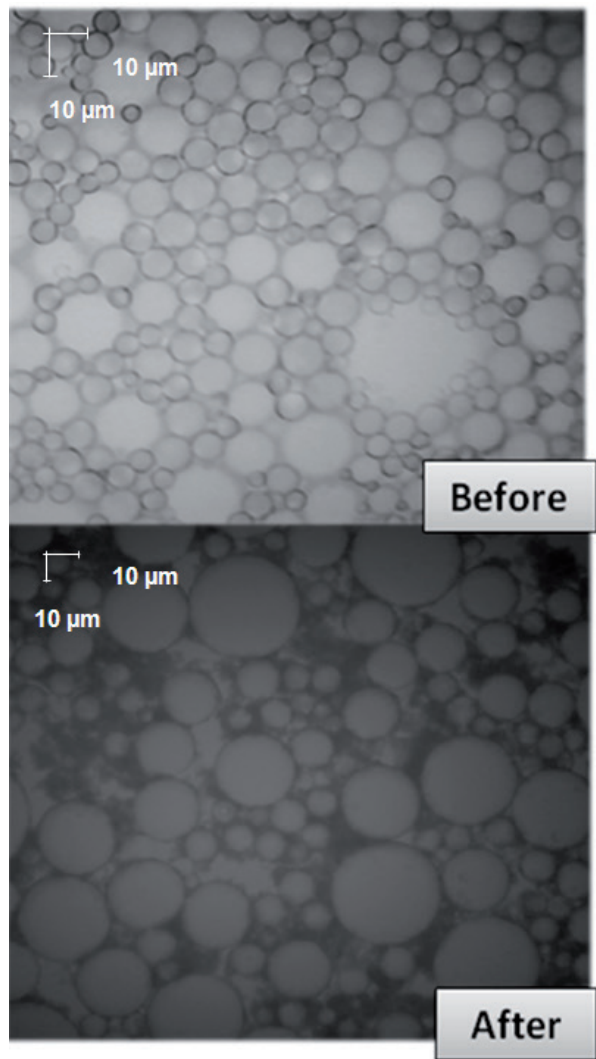

Fig. 5 Microscopic image of the samples before and after storage 


\section{Conclusions}

The present research work focused on the basic research of microcapsule production by membrane emulsification technology in bench scale. From the analysis of the experimental results, maltodextrin is considered as a suitable carrier material for emulsification process. Furthermore, it was observed that average particle size of synthesized microcapsule is $6.9 \mu \mathrm{m}$. After removal of water, synthesized final product was highly viscous, stable, white paste. Stability analysis result of synthesized microcapsule portrayed that they are stable at least 6 weeks also.

In the present investigation authors tried their best to establish the technology for microcapsule production by membrane based route (membrane emulsification technology). Authors believe that although the present research is still in infant stage but it will open a new arena in both academic and industrial (food and biotechnological) sector.

\section{References}

[1] Heinzen, C. "Microencapsulation solve time dependent problems for foodmakers." European Food and Drink Review. 3 (1). pp. 27-30. 2002.

[2] Madene, A., Jacquet, M., Scher, J., Desobry, J. "Flavour encapsulation and controlled release - a review." International Journal of Food Science and Technology. 41 (1). pp. 1-21. 2006.

DOI: 10.1111/j.1365-2621.2005.00980.x

[3] Tyagi, V. V., Kaushik, S. C., Tyagi, S. K., Akiyama, T. "Development of phase change materials based microencapsulated technology for buildings: a review." Renewable and Sustainable Energy Reviews. 15 (2). pp. 1373-1391. 2011. DOI: 10.1016/j.rser.2010.10.006
[4] Vladisavljevic, G., Williams, R. A. "Recent developments in manufacturing emulsions and particulate products using membranes." Advances in Colloid and Interface Science. 113 (1). pp. 1-20. 2005.

DOI: $10.1016 /$ j.cis.2004.10.002

[5] Wu, J., Fan, Q., Xia, Y., Ma, G. "Uniform-sized particles in biomedical field prepared by membrane emulsification technique." Chemical Engineering Science. 125 (1). pp. 85-97. 2015.

DOI: $10.1016 /$ j.ces.2014.08.016

[6] Khayata, N., Abdelwahed, W., Chehna, M. F., Charcosset, C., Fessi, H. "Stability study and lyophilization of vitamin E-loaded nanocapsules prepared by membrane contactor." International Journal of Pharmaceutics. 439 (1-2). pp. 254-259. 2012. DOI: 10.1016/j.ijpharm.2012.09.032

[7] Koc, M., Gungor, O., Zungur, A., Yalcin, B., Selek, I., Ertekin, F. K., Otles, S. "Microencapsulation of Extra Virgin Olive Oil by Spray Drying: Effect of Wall Materials Composition, Process Conditions, and Emulsification Method." Food and Bioprocess Technology. 8 (1). pp. 301-308. 2015. DOI: 10.1007/s11947-014-1404-9

[8] Ramakrishnan, S., Ferrando, M., Acena-Munoz, L., De Lamo-Castellví, S., Guell, C. "Fish Oil Microcapsules from O/W Emulsions Produced by Premix Membrane Emulsification." Food Bioprocess Technology. 6 (1). pp. 3088-3101. 2013. DOI: 10.1007/s11947-012-0950-2

[9] Malvern Instruments Ltd., Zetasizer Nano Series User Manual. [Online] Available from: http://www.biophysics.bioc.cam.ac.uk/files/Zetasizer Nano_user_manual_Man0317-1.1.pdf[Accessed: 8th July 2015]

[10] Joscelyne, S. M, Tragardh, G. "Membrane emulsification - a literature review." Journal of Membrane Science. 169 (1). pp. 107-117. 2000. DOI: 10.1016/S0376-7388(99)00334-8 